\title{
Hybrid Multi-Objective Network Planning Optimization Algorithm
}

\author{
Ning Liu ${ }^{12}$, David Plets ${ }^{2}$, Wout Joseph ${ }^{2}$ and Luc Martens ${ }^{2}$
}

\begin{abstract}
In this paper, a novel hybrid algorithm for the optimization of indoor wireless network planning is applied to a polyvalent arts centre. The results of the algorithm are compared with those of a heuristic network planner for three scenarios. Results show that our algorithm is effective for optimization of wireless networks, satisfying maximum coverage, minimal power consumption, minimal cost, and minimal human exposure.
\end{abstract}

\section{Introduction}

When planning wireless networks, different characteristics of the result can be considered and optimized, e.g. coverage, energy consumption, exposure and cost. In [5], energy conservation techniques on different types of base stations were compared. Exposure in office environments has been investigated in [8] and [21]. As for wireless network planning optimization with four main requirements, in [18, 20], researchers have focused on femtocells and hybrid (DVB-H/UMTS) networks, since these networks are associated with improved coverage and lower exposure. Plets et al. have presented a heuristic to optimize the exposure in indoor wireless networks, which is named the WiCa Heuristic Indoor Propagation Prediction (WHIPP) tool $[16,14,15]$.

\footnotetext{
${ }^{1}$ Ning Liu

School of Computer Science and Engineering, University Electronic Science and Technology of China, Chengdu, China

e-mail: ning.liu@intec.ugent.be

${ }^{2}$ Ning Liu · David Plets · Wout Joseph · Luc Martens

WiCa, Ghent University / iMinds, Dept. of Information Technology, Gaston Crommenlaan 8 box 201, B-9050 Ghent, Belgium,

e-mail: \{ning.liu, david.plets, wout.joseph, luc.martens $\} @$ intec.ugent.be
} 
Mainly three types of optimization algorithms are considered when optimizing indoor wireless environments planning [22, 13, 23]: PSO (Particle Swarm Optimization) [8], ACO (Ant Colony Optimization) [21] and GA (Genetic Algorithm) [18]. In [11], researchers use ACO to optimize the wireless networks in order to achieve coverage in energy-efficient way. In [3], Chen proposed an altered version of the PSO algorithm to solve the network planning problem in RFID systems. GAs have been developed to plan wireless communication networks in $[9,24]$ and have also shown good performance for coverage optimization and exposure minimization in $[10,2]$. GA and PSO algorithms have both yielded successful results and fast convergence in this field $[22,23]$, while ACO needs much more iterations for optimizing wireless network in [23].

In [12], a hybrid algorithm (combining GA and quasi-PSO) was proposed for the optimization of the wireless network planning, accounting for four requirements: maximum coverage, minimal power consumption, minimal cost, and minimal human exposure. In this paper, this algorithm and the WHIPP algorithm will be applied to a polyvalent arts centre for three different optimization scenarios. Section 2 briefly introduces the configuration and the fitness functions that are used. In Section 3, three scenarios are presented. A summary of our hybrid algorithm is provided in Section 4. The results and comparison with WHIPP of these scenarios for the indoor environment are provided in Section 5. Conclusions are presented in Section 6

\section{Configuration and Fitness Function}

\subsection{Configuration}

Fig. 1 shows a map of the ground floor of the Vooruit cultural centre (a polyvalent arts center). It is mainly constructed with large concrete walls and glass. The goal is to design a wireless network with $\mathrm{WiFi}(801.11 \mathrm{n})$ access points operating at a frequency of $2.4 \mathrm{GHz}$, with an antenna gain of $2 \mathrm{dBi}$, and for required received power of $-68 \mathrm{dBm}$ (for HD video coverage). The EIRP (Effective Isotropic Radiated Power) range of the access points runs from 0 to $20 \mathrm{dBm}$. The receiver antenna gain is $0 \mathrm{~dB}$. There are 202 possible positions to place WiFi access points; these are also the receiver points for which coverage and exposure will be calculated. The path loss PL (the ratio of the transmitted power and the received power) will be modeled according to the following two models.

- The first model is the two-slope model proposed by the IEEE 802.11 TGn channel models group [7].

$$
\begin{array}{r}
P L(d)=P L_{\text {free }}(d)+X\left(d \leq d_{b r}\right) \\
P L(d)=P L_{\text {free }}(d)+32 \log _{10}\left(\frac{d}{d_{b r}}\right)+X\left(d>d_{b r}\right)
\end{array}
$$




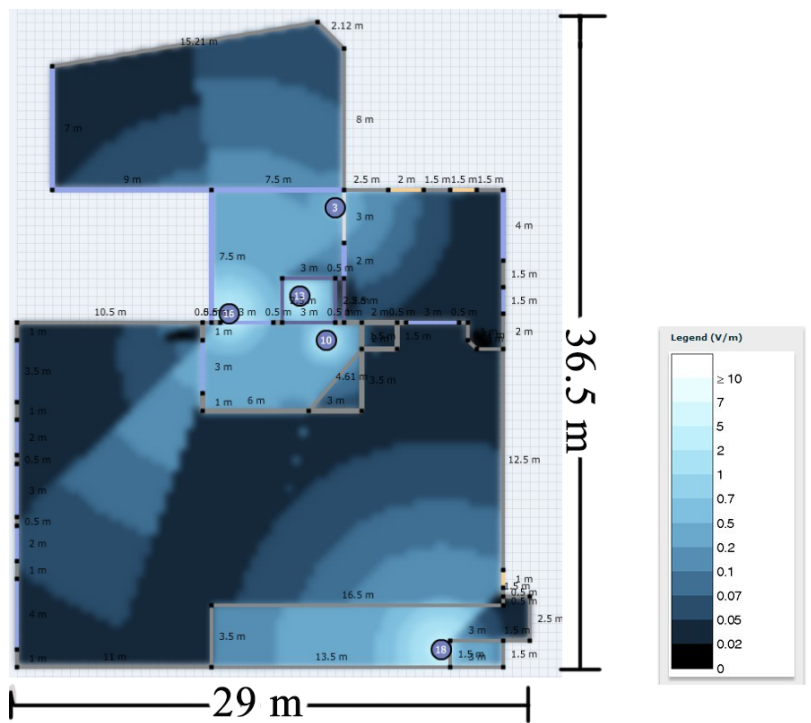

Fig. 1 Map of The Indoor Environment and the Exposure Level for Scenario III for the SIDP Model

Where $P L_{\text {free }}(d)$ is the free space loss [19]. The variation of path loss $X$ due to shadowing follows a lognormal distribution, with two different standard deviations $\sigma[\mathrm{dB}]$ of $X$ for $d \leq d_{b r}$ and $d>d_{b r}$. In this situation, parameters are considered as follows: $d_{b r}$ of $10 m, \sigma=3 d B$ for $d \leq d_{b r}$ and $\sigma=5 d B$ for $d>d_{b r}$, corresponding to a $95 \%$ shadowing margin of $4.92 d B$ and $8.2 d B$ for $d \leq d_{b r}$ and $d>d_{b r}$ respectively [7]. The temporal fading margin is set at $5 d B$ [1].

- The second model is Simple Indoor Dominant Path Loss model used in [17]. The shadowing margin is set at $7 d B(95 \%)$ and the fading margin at $5 d B(99 \%)$.

\subsection{Fitness Functions}

Four different fitness functions will be investigated for the optimization of the network planning. Each fitness function optimizes one or more of the four main wireless network characteristics (coverage, power consumption, cost, human exposure). The results of the different functions, $f_{i}(i=1,2,3)$ will range from 0 to 100 , so that they have an equal contribution when they are combined in a new fitness function (see Section 2.2.4). A comparable value of the weights $\left(w_{1}, w_{2}, w_{3}\right)$ of the different functions $\left(f_{1}, f_{2}, f_{3}\right)$ then causes a comparable influence of the function on the combined fitness function $\left(f_{4}\right)$. 


\subsubsection{Coverage}

The first fitness function represents coverage fitness as in Eq. (2),

$$
f_{1}=100 \frac{f_{\text {sol }}}{f_{\text {tot }}}
$$

Where $f_{\text {tot }}$ is the number of all reception points (202 for the considered building), $f_{\text {sol }}$ is the number of reception points covered by the current solution in this indoor environment and $f_{1}$ represents the coverage percentage of the considered network configuration.

\subsubsection{Power Consumption and Economic Cost}

In Eq. (3), $f_{2}$ represents the ratio of the actual power consumption of the considered network configuration to the maximum achievable power consumption in the network:

$$
f_{2}=100 \frac{\sum_{i=1}^{n} p_{i}}{p_{\max }},
$$

where $p_{i}$ is the power consumption of the $\mathrm{i}$-th access point ( $12 \mathrm{~W}$ for a WiFi access point which is on [6], $0 \mathrm{~W}$ when it is turned off), $p_{\max }$ is the total power consumption when all possible access points are turned on. The actual EIRP also affects the total power consumption. However, because the impact is small [6], we neglect the effect of the radiated power and assume a fixed value of $12 \mathrm{~W}$ per access point $[4,6]$. Eq. (3) then reduces to

$$
f_{2}=100 \frac{m}{n},
$$

where $m$ is the number of access points which are turned on, and $n$ total number of possible positions (202 for Vooruit).

The cost of all installed access points represents the economic cost (Capital Expenditures). Since a fixed power consumption is assumed for all access points, $f_{2}$ represents both the economic cost fitness function and the power consumption fitness function of the considered network deployment.

\subsubsection{Exposure}

In Eq. (5), $f_{3}$ is a fitness function based on the median electric-field strength $E_{m}[\mathrm{~V} / \mathrm{m}]$ observed at the considered receiver points in the environment.

$$
f_{3}=100 \frac{E_{m}}{E_{\max }},
$$

where $E_{\max }$ is the maximal median electric-field value that could be achieved. This is the case when all (202) access points are turned on with an EIRP of $20 \mathrm{dBm}$, 
yielding a value for $E_{\max }$ of $2.19 \mathrm{~V} / \mathrm{m}$ is obtained for TGn model and $2.46 \mathrm{~V} / \mathrm{m}$ for SIDP model. The optimal solution of this fitness function has a minimal median electric field strength.

\subsubsection{Combined Fitness Function}

In Eq. (6), $f_{4}$ is a global fitness function which combines above three presented fitness function:

$$
f_{4}=w_{1} f_{1}-w_{2} f_{2}-w_{3} f_{3}
$$

where $w_{i}$ is the weight (values between 0 and 1) of function $f_{i}$ with its value determined by the importance of $f_{i}$. By adjusting $w_{i}$, four demands can be jointly optimized. 2. $w_{2}$ and $w_{3}$ are chosen so that coverage is the most important factor in optimization $\left(w_{1}=1\right)$. However, on top of coverage optimization, energy consumption $\left(w_{2}\right)$ and exposure $\left(w_{3}\right)$ are also important, but less than coverage. The values of w2 and w3 need to be small enough to obtain a solution with $100 \%$ coverage, but large enough to still minimize energy consumption and exposure. Consequently, when we increase w2, results with less access points are expected. When we increase $w_{3}$, results with lower exposure levels are expected. The weights control the value of the fitness function and the fitness value affects the result of the algorithm. The best solutions are the ones with the highest combined fitness function values, as they correspond to higher coverage rates, lower total power consumptions (and cost), and lower exposure values. For the optimization of the fitness function, a hybrid genetic optimization algorithm is used [12].

\section{Scenarios}

We define three scenarios to investigate the influence of coverage, exposure, and cost restrictions on the network deployment for Vooruit (in Fig. 1) by applying our algorithm and comparing with the WHIPP algorithm. Unlike for our hybrid optimization algorithm, the WHIPP optimization is not based on the use of a fitness function and the evaluation of a number of iterations. It builds a solution based on a number of optimization phases following a fixed procedure. The WHIPP algorithm allows an optimization for $100 \%$ coverage with a minimal number of APs, as well as an optimization for $100 \%$ coverage with a minimal exposure. This allows a comparison with the output of Scenarios I and II by our algorithm, as described hereafter. All scenarios are applied to the configuration and using the PL model of Section 2. 


\subsection{Scenario I: Coverage with minimal number of APS}

Scenario I aims to obtain $100 \%$ coverage rate with a minimal number of access points (minimal both cost and power consumption). We select the weight $w_{2}$ for the $f_{2}$ as 0.2 , since this value is large enough to minimize the number of APs and small enough to aim for a coverage rate of $100 \%$. The combined fitness function of Eq. (6) in scenario $\mathrm{I}$ is as follows:

$$
f_{4}=f_{1}-0.2 f_{2} \quad\left(w_{1}=1, w_{2}=0.2\right)
$$

\subsection{Scenario II: Coverage with minimal human exposure}

Scenario II intends to obtain $100 \%$ coverage rate with a minimal median exposure. The combined function $f_{4}$ is as follows:

$$
f_{4}=f_{1}-0.2 f_{3} \quad\left(w_{1}=1, w_{3}=0.2\right)
$$

We select the weight for the exposure level fitness $w_{3}$ as 0.2 , since this value is large enough to minimize the exposure level and small enough to obtain $100 \%$ coverage.

\subsection{Scenario III: Coverage with minimal human exposure and minimal number of APs}

Scenario III is defined to consider a tradeoff among a high coverage rate, a low total power consumption and a low median electric-field strength. Under the condition of scenario III in Eq. (9), we consider different requirements together: coverage, number of access points (cost and power consumption), and exposure level.

$$
f_{4}=f_{1}-0.2 f_{2}-0.2 f_{3} \quad\left(w_{1}=1, w_{2}=0.2, w_{3}=0.2\right)
$$

\section{Our Algorithm}

Fig. 2 shows the flow chart that corresponds to the operation of our algorithm. The main operations of the genetic algorithm are crossover and mutation operations.

Firstly, 1000 random solutions are generated and their fitness values are calculated. The solutions with the top-80 fitness values are put into a list.

Secondly, after sorting this solution list based on their fitness values, the top-40 of the list with the high fitness values is called 'good list' and the rest of the list is called 'bad list'. 
Thirdly, new solutions are generated by a crossover operation between a father solution from the good list and a mother solution from the bad list. In this operation one third of the father solution is combined with two third of a mother solution. If the offspring gets a higher fitness value than that of mother solution, we put it into the corresponding location of the list.

Fourthly, the mutation operation adds random changes in a solution and makes the algorithm converge to a global optimum instead of to a local optimum. During each mutation, a solution has equal possibility to perform one of the following operations:

- Turn off one access point in this solution;

- Turn on an access point with random power value;

- Turn on an access point with random power value and turn off another access point of this solution;

- Turn off two access points of this solution and turn on an access point with random power value;

- Change the power value of an access point of this solution;

- Change the position of an access point of this solution.

GAs and PSOs are suitable to solve the multi-objective problem described in Section 1. Since we can obtain benefit from the evaluation and heredity of GA, the GA is better than PSO. PSO performs better, when the solution consists of only one AP, due to a slight change of solution in each iteration is better to quickly find the optimal solution. Therefore, our algorithm introduces operations of PSOs into the GA system. When only one access point is sufficient, offspring are generated by using the quasi-PSO with a certain probability. The new algorithm approaches the global optimum more efficiently.

\section{Results}

\subsection{Simple Indoor Dominant Path Loss Model (SIDP)}

The results for the scenarios described above are investigated for WHIPP and our algorithm based on the SIDP model and are listed in Table 1. For all scenarios, the coverage of all methods is $100 \%$. For scenario I, our algorithm obtains a solution with 3 access points, while WHIPP obtains a solution with 4 access points. The solution of our algorithm generates a lower median exposure level of $123.7 \mathrm{mV} / \mathrm{m}$ versus $155.6 \mathrm{mV} / \mathrm{m}$ of WHIPP, due to the lower number and EIRP of APs of the solution of our algorithm. The solution of our algorithm for scenario II also generates a slightly lower median exposure level (9.3\% lower) than that of WHIPP, although, the $95 \%$ percentile exposure level of our algorithm is much higher than that of WHIPP, since there is less spatial homogeneity in the exposure levels of our solution. The solution of our algorithm for scenario III is a compromise between all criteria (high coverage, low exposure and needs a low number of APs). It shows the 


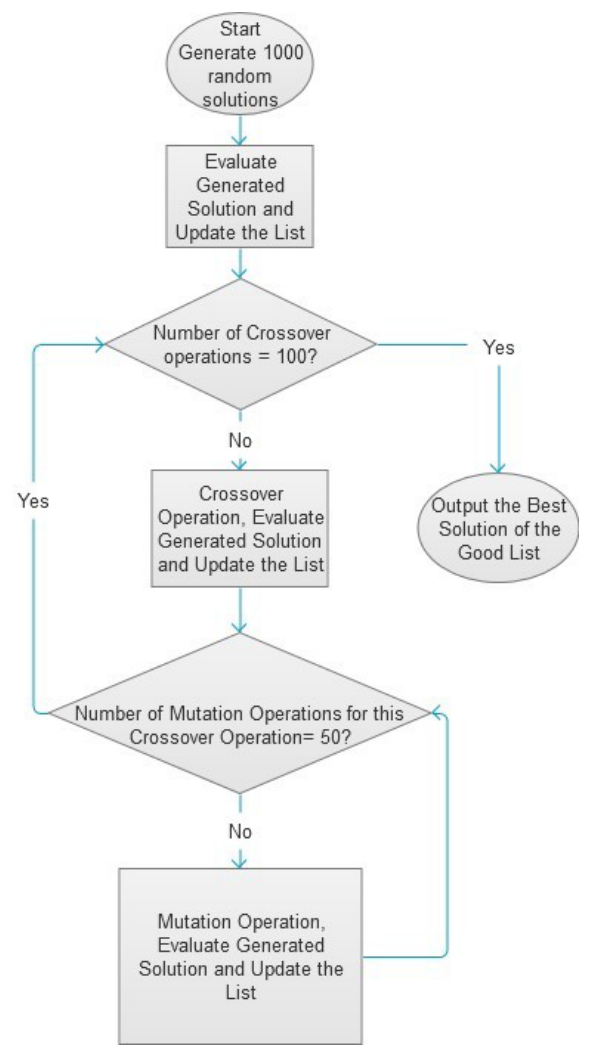

Fig. 2 Flow Chart of Our Algorithm

advantage of our algorithm, since scenario III is difficult to implement in the WHIP$\mathrm{P}$ tool. The solution of our algorithm for scenario III requires 5 APs and generates a median exposure level of only $47.2 \mathrm{mV} / \mathrm{m}$ which is about $1 \%$ higher than that of scenario II which needs 10 APs. Fig. 1 shows the electronic field distribution for scenario III in the considered building. The location and EIRP of the APs is also indicated. Compared to WHIPP [15, 14], the simulation time (last column in Table 1) of the our algorithm is always much higher than that of WHIPP, since WHIPP is a heuristic. Limiting the simulation time of our algorithm to the WHIPP simulation times would yield worse results, since a substantial number of iterations is required for this type of algorithms (GAs). However, since network planning is mostly a task that is performed only once, large computation times are not really an issue if the algorithm finally provides a better result.

Fig. 3 shows the comparison of CDF of the exposure values based on the SIDP model for WHIPP and our algorithm. It shows that the exposure level of our algorithm is always lower than that of WHIPP at the same probability for scenario I. However, when we consider scenario II, this situation is reversed when the probability greater than $80 \%$ (see Fig. 3), since the less spatial homogeneity in the exposure 
Table 1 The Results of Scenarios for indoor Environment Based on Simple Indoor Dominant Path Loss Model

\begin{tabular}{|c|c|c|c|c|c|c|c|}
\hline Scenarios & Method & $\begin{array}{l}\text { Cove } \\
\text { Rate } \\
{[\%]}\end{array}$ & APs [-] & $\begin{array}{l}E_{50}{ }^{a} \\
{[m V / m]}\end{array}$ & $\begin{array}{l}E_{95^{b}} \\
{[m V / m]}\end{array}$ & $\operatorname{EIRP}[d B m]$ & $\begin{array}{l}\text { Simulation } \\
\text { Time [s] }\end{array}$ \\
\hline \multirow[t]{2}{*}{ Scenario I } & WHIPP & 100 & 4 & 155.6 & 819.7 & $4 \times 20 d B m$ & 111 \\
\hline & Our Algorithm & 100 & 3 & 123.7 & 775.0 & $15 \mathrm{dBm}, 2 \times 20 \mathrm{dBm}$ & $8.8 \times 10^{3}$ \\
\hline \multirow[t]{2}{*}{ Scenario II } & WHIPP & 100 & 12 & 51.6 & 190.2 & $\begin{array}{l}-26 d B m,-13 d B m,-1 d B m, \\
0 d B m, 5 \times 1 d B m, 2 d B m, \\
4 d B m, 5 d B m\end{array}$ & 274 \\
\hline & Our Algorithm & 100 & 10 & 46.8 & 422.2 & $\begin{array}{l}2 \times 0 d B m, \quad 2 d B m, \quad 4 d B m \\
2 \times 5 d B m, 3 \times 9 d B m, 17 d B m\end{array}$ & $7.2 \times 10^{4}$ \\
\hline \multirow{2}{*}{ Scenario III } & $\mathrm{WHIPP}^{c}$ & - & - & - & - & - & - \\
\hline & Our Algorithm & 100 & 5 & 47.2 & 465.3 & $\begin{array}{l}3 \mathrm{dBm}, \quad 10 \mathrm{dBm} \\
16 \mathrm{dBm}, 18 \mathrm{dBm}\end{array}$ & $6.6 \times 10^{4}$ \\
\hline
\end{tabular}

${ }^{a} E_{50}: 50 \%$ percentile of $\mathrm{E}(\mathrm{mV} / \mathrm{m})$

${ }^{b} E_{95}: 95 \%$ percentile of $\mathrm{E}(\mathrm{mV} / \mathrm{m})$

${ }^{c}$ WHIPP cannot optimize 3 requirements as required for scenario III

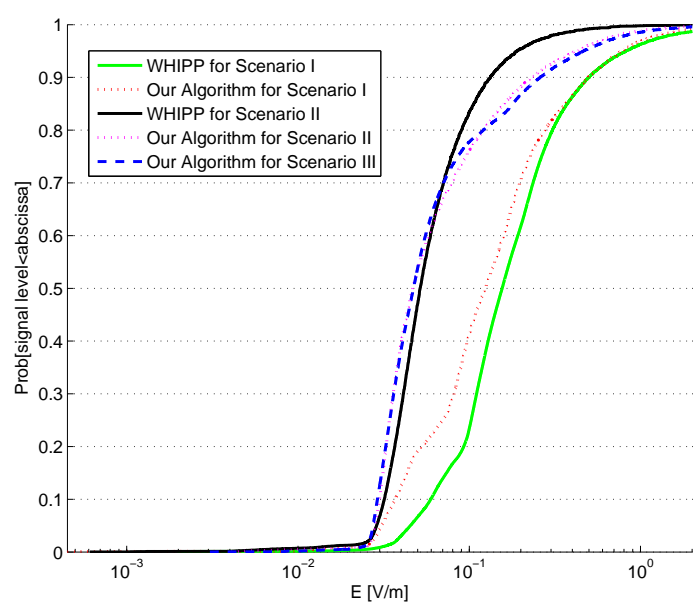

Fig. 3 Comparison The CDF of The Exposure Results for Indoor Environment (Based on The Simple Indoor Dominant Path Loss Model)

levels of our solution. The exposure level of our algorithm for scenario III is very close to that of our algorithm for scenario II. 
Table 2 The Results of Scenarios for Indoor Environment Based on TGn Two-Slope Path Loss Model

\begin{tabular}{|c|c|c|c|c|c|c|c|}
\hline Scenarios & Method & $\begin{array}{l}\text { Cove } \\
\text { Rate } \\
{[\%]}\end{array}$ & Ps [-] & $\begin{array}{l}E_{50}{ }^{a} \\
{[m V / m]}\end{array}$ & $\begin{array}{l}E_{95}{ }^{b} \\
{[m V / m]}\end{array}$ & $\operatorname{EIRP}[d B m]$ & $\begin{array}{l}\text { Simulation } \\
\text { Time }[\mathrm{s}]\end{array}$ \\
\hline \multirow[t]{2}{*}{ Scenario I } & WHIPP & 100 & 2 & 164.1 & 631.8 & $2 \times 20 d B m$ & 1 \\
\hline & Our Algorithm & 100 & 2 & 115.5 & 434.9 & $8 d B m, 19 d B m$ & 35 \\
\hline \multirow[t]{2}{*}{ Scenario II } & WHIPP & 100 & 6 & 35.0 & 116.1 & $6 \times 1 \mathrm{dBm}$ & 6 \\
\hline & Our Algorithm & 100 & 5 & 34.5 & 118.6 & $2 \times 1 \mathrm{dBm}, 3 \times 2 \mathrm{dBm}$ & 137 \\
\hline \multirow[t]{2}{*}{ Scenario III } & $\mathrm{WHIPP}^{c}$ & - & - & - & - & - & - \\
\hline & Our Algorithm & 100 & 4 & 41.6 & 294.0 & $1 \mathrm{dBm}, 2 \times 2 \mathrm{dBm}, 18 \mathrm{dBm}$ & 104 \\
\hline
\end{tabular}

${ }^{a} E_{50}: 50 \%$ percentile of $\mathrm{E}(\mathrm{mV} / \mathrm{m})$

${ }^{b} E_{95}: 95 \%$ percentile of $\mathrm{E}(\mathrm{mV} / \mathrm{m})$

${ }^{c}$ WHIPP cannot optimize 3 requirements as required for scenario III

\subsection{TGn Model}

Table 2 lists the results of WHIPP and our algorithm. As for scenario I, WHIPP and our algorithm both obtain a solution with 2 APs. The median and the $95 \%$ percentile exposure levels of our algorithm for scenario I are both lower than that of WHIPP, due to the lower EIRP of the APs of our algorithm. The differences between the exposure results of WHIPP and our algorithm for scenario II is small. The solution of our algorithm needs 6 APs, while that of WHIPP needs 5 APs. For the exposure level for scenario II, the median exposure level of WHIPP is $1.5 \%$ higher than that of our algorithm. However, $E_{95}$ of our algorithm is $2.1 \%$ higher than that of WHIPP. For scenario III (Table 2), our algorithm obtains a solution with $4 \mathrm{APs}(20 \%$ lower than that of our algorithm for scenario II) and generates a median exposure of $41.6 \mathrm{mV} / \mathrm{m}$ (74.6\% lower than that of our algorithm for scenario I). As for the simulation time, that of WHIPP is again always lower than that of our algorithm for each scenario, but calculation times are limited for a algorithm as well ( maximum $=137 \mathrm{~s}$ for scenario III).

Comparison of the CDFs for the TGn model shows that the exposure values for our algorithm are mostly lower than for WHIPP at the same probability for scenario I (see Fig.4). The difference between the exposure levels of WHIPP and that of our algorithm for scenario II is small. For scenario III, the curve of our algorithm is between scenario I (minimal cost or number of APs) and scenario II (minimal exposure).

\section{Conclusions}

A hybrid genetic optimization algorithm has been proposed to optimize coverage rate, human exposure to radio-frequency sources, energy consumption and econom- 


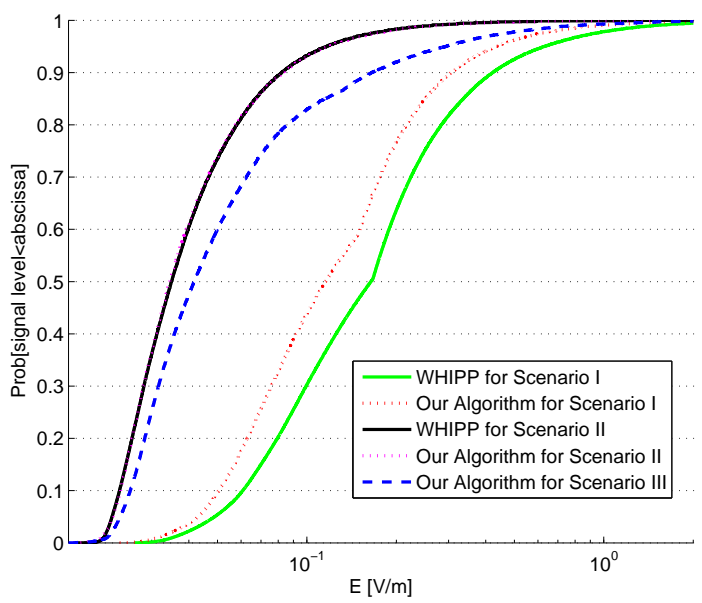

Fig. 4 Comparison The CDF of The Exposure Results for Indoor Environment (Based on The TGn Model)

ic cost of the indoor wireless networks. Specific fitness functions were used to evaluate the solutions for a homogeneous WiFi network. Three scenarios are defined to verify the performance of the algorithm and good results are obtained. An application for a realistic indoor environment (Vooruit) is investigated leading to reductions of cost and exposure when applying our algorithm compared to a heuristic tool (a median exposure level reduction of $9 \%$ or a cost reduction of $25 \%$ are obtained compared to WHIPP based on the SIDP model). Future research enable planning of heterogeneous wireless networks for various indoor environments.

\section{References}

1. Bultitude, R.J.C.: Measurement, characterization and modeling of indoor $800 / 900 \mathrm{mhz}$ radio channels for digital communications. Communications Magazine, IEEE 25(6), 5-12 (1987). DOI 10.1109/MCOM.1987.1093629

2. Cerri, G., De Leo, R., Micheli, D., Russo, P.: Base-station network planning including environmental impact control. Communications, IEE Proceedings- 151(3), 197-203 (2004). DOI 10.1049/ip-com:20040146(410) 151

3. Chen, H., Zhu, Y., Hu, K., Ku, T.: RFID network planning using a multi-swarm optimizer. Journal of Network and Computer Applications 34(3), 888 - 901 (2011). DOI 10.1016/j.jnca.2010.04.004

4. Deruyck, M., Joseph, W., Lannoo, B., Colle, D., Martens, L.: Designing Energy-Efficient Wireless Access Networks: LTE and LTE-Advanced. Internet Computing, IEEE 17(5), 39-45 (2013). DOI 10.1109/MIC.2013.6

5. Deruyck, M., Vereecken, W., Joseph, W., Lannoo, B., Pickavet, M., Martens, L.: Reducing the power consumption in wireless access networks: overview and recommendations. PROGRESS IN ELECTROMAGNETICS RESEARCH-PIER 132, 255-274 (2012) 
6. Deruyck, M., Vereecken, W., Joseph, W., Lannoo, B., Pickavet, M., Martens, L.: Reducing the power consumption in wireless access networks: overview and recommendations. Progress In Electromagnetics Research 132, 255-274 (2012). DOI 10.2528/PIER12061301

7. Erceg, V., Schumacher, L., et al.: IEEE P802. 11 Wireless LANs. TGn Channel Models, doc.: IEEE pp. 802-11

8. Joseph, W., Verloock, L., Goeminne, F., Vermeeren, G., Martens, L.: Assessment of general public exposure to LTE and RF sources present in an urban environment. BIOELECTROMAGNETICS 31, 576 - 579 (2010)

9. Jourdan, D., de Weck, O.: Layout optimization for a wireless sensor network using a multiobjective genetic algorithm. In: Vehicular Technology Conference, 2004. VTC 2004-Spring. 2004 IEEE 59th, vol. 5, pp. 2466-2470 Vol.5 (2004). DOI 10.1109/VETECS.2004.1391366

10. Koutitas, G., Samaras, T.: Exposure minimization in indoor wireless networks. Antennas and Wireless Propagation Letters, IEEE 9, 199-202 (2010). DOI 10.1109/LAWP.2010.2045870

11. Lee, J.W., Choi, B.S., Lee, J.J.: Energy-efficient coverage of wireless sensor networks using ant colony optimization with three types of pheromones. Industrial Informatics, IEEE Transactions on 7(3), 419-427 (2011). DOI 10.1109/TII.2011.2158836

12. Liu, N., Plets, D., Goudos, S., Joseph, W., Martens, L.: Multi-objective network planning optimization algorithm: Human exposure, power consumption, cost, and coverage. Wireless Communications and Mobile Computing, submitted

13. Nagy, L.: Indoor radio coverage optimization for WLAN. In: 2nd European Conference on Antennas and Propagation (EuCAP 2007), p. 225 (2007). DOI 10.1049/ic.2007.1348

14. Plets, D., Joseph, W., Vanhecke, K., Martens, L.: A heuristic tool for exposure reduction in indoor wireless networks. In: Antennas and Propagation Society International Symposium (APSURSI), 2012 IEEE, pp. 1-2 (2012). DOI 10.1109/APS.2012.6348505

15. Plets, D., Joseph, W., Vanhecke, K., Tanghe, E., Martens, L.: Development of an accurate tool for path loss and coverage prediction in indoor environments. In: Antennas and Propagation (EuCAP), 2010 Proceedings of the Fourth European Conference on, pp. 1-5 (2010)

16. Plets, D., Joseph, W., Vanhecke, K., Tanghe, E., Martens, L.: Coverage prediction and optimization algorithms for indoor environments. EURASIP Journal on Wireless Communications and Networking, Special Issue on Radio Propagation, Channel Modeling, and Wireless, Channel Simulation Tools for Heterogeneous Networking Evaluation 1 (2012)

17. Plets, D., Joseph, W., Vanhecke, K., Tanghe, E., Martens, L.: Simple indoor path loss prediction algorithm and validation in living lab setting. Wireless Personal Communications 68(3), 535-552 (2013). DOI 10.1007/s11277-011-0467-4

18. Ran, M., Ezra, Y.B.: Green femtocell based on uwb technologies. Novel Applications of the UWB Technologies, August pp. 175-194 (2011)

19. Saunders, S.R.: Antennas and Propagation for Wireless Communication Systems. John Wiley \& Sons Ltd (1999)

20. Unger, P., Schack, M., Kurner, T.: Minimizing the Electromagnetic Exposure Using Hybrid (DVB-H/UMTS) Networks. Broadcasting, IEEE Transactions on 53(1), 418-424 (2007). DOI 10.1109/TBC.2006.889207

21. Verloock, L., Joseph, W., Vermeeren, G., Martens, L.: Procedure for assessment of general public exposure from wlan in offices and in wireless sensor network testbed. HEALTH PHYSICS 98, 628 - 638 (2010)

22. Vilovic, I., Burum, N., Sipus, Z.: Design of an indoor wireless network with neural prediction model. In: Antennas and Propagation, 2007. EuCAP 2007. The Second European Conference on, pp. 1-5 (2007)

23. Vilovic, I., Burum, N., Sipus, Z.: Ant colony approach in optimization of base station position. In: Antennas and Propagation, 2009. EuCAP 2009. 3rd European Conference on, pp. 28822886 (2009)

24. Yun, Z., Lim, S., Iskander, M.: An integrated method of ray tracing and genetic algorithm for optimizing coverage in indoor wireless networks. Antennas and Wireless Propagation Letters, IEEE 7, 145-148 (2008). DOI 10.1109/LAWP.2008.919358 